\title{
Response of Varieties and Integrated Nutrient Management on Economic Parameters of Isabgol (Plantago ovata Forsk.) under Northern Dry Zone of Karnataka
}

\author{
Siddalingayya V. Salimath", K. N. Kattimani, Y. K. Kotikal, N. S. Nagarja, \\ D. R. Patil, Jameel Jhalegar and J. Venkatesh \\ College of Horticulture, University of Horticultural Sciences, Bagalkot, India \\ *Corresponding author
}

\author{
A B S T R A C T
}

\begin{tabular}{l} 
K e y w o r d s \\
$\begin{array}{l}\text { Gujarat Isabgol-2, } \\
\text { Growth, Vallabh } \\
\text { Isabgol-1, Yield }\end{array}$ \\
Article Info \\
$\begin{array}{l}\text { Accepted: } \\
22 \text { November } 2020 \\
\text { Available Online: } \\
10 \text { December } 2020\end{array}$ \\
\hline
\end{tabular}

\section{Ke y w o r d s}

Gujarat Isabgol-2,

Growth, Vallabh

, Yield

\section{Introduction}

According to the Ministry of AYUSH, Government of India, medicinal plants raw material is estimated as international commodity worth of Rs. 80-90 billion.

The export value of medicinal plants and related products in India is approximately Rs. 110 billion. In 2012-13, the export value of AYUSH products was Rs. 24,741 crores.

The percentage share of AYUSH products in the total trade of India in 2013-2014 was 0.36

\begin{abstract}
The field experiment was conducted to evaluate the performance of yield parameters of two Isabgol cultivars for commercial production in northern dry zone of Karnataka during two years 2015-16 and 2016-17. The experiment was laid out in split plot design (SPD) with sixteen INM treatment combinations at the college of horticulture, Bagalkot. Analysis of both years with pooled data exhibited higher value was recorded in Vallabh Isabgol-1in conomic parameters viz., gross return (Rs), net return (Rs), B;C ratio, as compared to Gujarat Isabgol-2. Whereas the higher values with INM treatments with respect to plant growth parameters were observed in Viz. $\mathrm{N}_{11}-75 \%$ RD of FYM $\left(7.5 \mathrm{t} \mathrm{ha}^{-1}\right)+75 \%$ RD of NPK $\left(37.5: 18.75: 22.50 \mathrm{~kg} \mathrm{ha}^{-1}\right)+$ Azospirillum $\left(5 \mathrm{~kg} \mathrm{ha}^{-1}\right)+P S B\left(3 \mathrm{~kg} \mathrm{ha}^{-1}\right)+\mathrm{ZnSO}_{4}(15 \mathrm{~kg}$ $\left.\mathrm{ha}^{-1}\right)+\mathrm{FeSO}_{4}\left(7.5 \mathrm{~kg} \mathrm{ha}^{-1}\right) v i z$. , net returns $\left(\mathrm{q} \mathrm{Rs}^{-1}\right)$, gross returns, B:C ratio $\left(1: 1 \mathrm{Rs} \mathrm{ha}^{-1}\right)$. Higher values for interaction effect on growth parameters were recorded in $\mathrm{V}_{1} \mathrm{~N}_{11}$. viz., seed yield $\left(15.50 \mathrm{q} \mathrm{ha}^{-1}\right)$, net returns $\left(\mathrm{q} \mathrm{Rs}^{-1}\right)$, gross returns $\mathrm{B}$ : C ratio $\left(\mathrm{Rs} \mathrm{ha}^{-1}\right)$.
\end{abstract}

per cent. The global market for herbal drugs is increasing in a steady manner and the global herbal trade will reach Rs. 7 trillion by 2050 . This indicates that, there is a vast scope for cultivation of medicinal plants (Sen and Chakraborty, 2017).

Approximately, 25,000 effective plant-based formulations are available in Indian system of medicine which is commonly used by rural and ethnic people in India and the popularity of such medicine is also increasing among the common people. It is estimated that more than 2000 tonnes of medicinal plants raw material 
is required annually. More than 1500 herbals are sold as dietary supplements or ethnic traditional medicines.

Nearly, 960 species of medicinal plants are in trade and among them 178 species have annual consumption level of 100 metric tonnes (Anon, 2015b).

The use of judicious combination of organic and inorganic fertilizers is not only essential to maintain soil health but also to sustain productivity as to get more than average national productivity of $0.54 \mathrm{q} \mathrm{ha}^{-1}$ (Goraya and Ved, 2019).

It can be improved more than thrice, by increase in productivity $\left(1.5 \mathrm{q} \mathrm{ha}^{-1}\right)$ a use of high yielding variety with an INM with good agronomic practice. Hence, the present investigation was undertaken.

\section{Gross return (Rs. ha $\left.{ }^{-1}\right)$}

The economics parameters influenced by varieties, integrated nutrient management and their interaction on gross return $\left(\right.$ Rs.h $\left.^{-1}\right)$, net returns $\left(\right.$ Rs.ha $\left.^{-1}\right)$ and benefit cost ratio of pooled data taken and are represented in Table 1.

The gross return (Rs. ha ${ }^{-1}$ ) was significantly influenced by varieties.

Maximum gross return (Rs. 73,273.24) was recorded in Vallabh Isabgol-1 as compared to Gujarat Isabgol-2 (Rs. 52,319.1) because of high seed yield produced by individual genotype leads to higher cost of cultivation, market prices in that cropping period leads to increase gross returns.

Gross return (Rs. ha ${ }^{-1}$ ) was significantly influenced by integrated nutrient management. Significantly maximum gross return (Rs. 82,472.6) was recorded with supply of $\mathrm{N}_{11}(75 \% \mathrm{RD}$ of FYM $+75 \%$ RD of $\mathrm{NPK}+$ Azospirillum $+P S B+\mathrm{ZnSO}_{4}+$ $\left.\mathrm{FeSO}_{4}\right)$ which was on par with $\mathrm{N}_{16}(50 \% \mathrm{RD}$ of FYM + $75 \%$ RD of NPK + Azospirillum + $P S B+\mathrm{ZnSO}_{4}+\mathrm{FeSO}_{4}$ ) (Rs. 77,499.7), $\mathrm{N}_{6}$ (Vermicompost $+50 \%$ RD of NPK + Azospirillum + Azotobacter $)(79,831.8)$ and $\mathrm{N}_{4}$ (RD of FYM + RD of NPK+ $\mathrm{ZnSO}_{4}+$ $\left.\mathrm{FeSO}_{4}\right)(\mathrm{Rs} .79,026.6)$. Minimum gross return (Rs. 50,105.6) was recorded with $\mathrm{N}_{13}(50 \%$ $\mathrm{RD}$ of FYM + $50 \%$ RD of NPK + Azotobacter).

This high gross return because of use of INM treatments leading to higher seed yield and cost of cultivation leads to more market prices (Hindiholi, 2006).

Gross return significantly influenced by varieties and integrated nutrient management. The maximum gross return (Rs.92,759.2) was recorded in Vallabh Isabgol-1 supplied with $75 \%$ RD of $\mathrm{FYM}+75 \% \mathrm{RD}$ of NPK + Azospirillum $+P S B+\mathrm{ZnSO}_{4}+\mathrm{FeSO}_{4}$ $\left(\mathrm{V}_{11} \mathrm{~N}_{11}\right)$ which was on par with Vallabh Isabgol-1, with supply of $50 \%$ RD of FYM + $75 \%$ RD of NPK + Azospirillum + PSB + $\mathrm{ZnSO}_{4}+\mathrm{FeSO}_{4}\left(\mathrm{~V}_{1} \mathrm{~N}_{16}\right)$ (Rs.91,683.5) as compared to other treatment combination.

Minimum gross return (Rs.40,502.5) was recorded Gujarat Isabgol-2 supplied with (50 $\%$ RD of FYM + $50 \%$ RD of NPK + Azotobacter $)\left(\mathrm{V}_{2} \mathrm{~N}_{13}\right)$.

\section{Net return (Rs. ha $\left.{ }^{-1}\right)$}

The pooled data significantly maximum net return (Rs. ha ${ }^{-1}$ ) (Rs. 52,526.6) was recorded in Vallabh Isabgol-1 as compared to (Rs. 48,420) Gujarat Isabgol-2.

Because of higher yield and more market prices fetched that period of seeds leads higher net returns. Similar results also reported by Charak et al., (2005). 
Table.1 Economics of Isabgol production as influenced by varieties and integrated nutrient management

\begin{tabular}{|c|c|c|c|c|c|c|c|c|c|c|c|c|c|c|c|c|c|c|c|c|c|c|c|c|c|c|c|}
\hline \multirow{3}{*}{$\begin{array}{c}\text { Varieties } \\
\text { Nutrients } \\
\text { Nutrients }\end{array}$} & \multicolumn{9}{|c|}{2015} & \multicolumn{9}{|c|}{2016} & \multicolumn{9}{|c|}{ Pooled data } \\
\hline & \multicolumn{3}{|c|}{ Gross return (Rs. ha ${ }^{-1}$ ) } & \multicolumn{3}{|c|}{ Net return (Rs. ha $\left.{ }^{-1}\right)$} & \multicolumn{3}{|c|}{ B:C ratio } & \multicolumn{3}{|c|}{ Gross return (Rs. ha- $\left.{ }^{-1}\right)$} & \multicolumn{3}{|c|}{ Net return (Rs. ha ${ }^{-1}$ ) } & \multicolumn{3}{|c|}{ B:C ratio } & \multicolumn{3}{|c|}{ Gross return (Rs. ha ${ }^{-1}$ ) } & \multicolumn{3}{|c|}{ Net return (Rs. ha' $\left.{ }^{-1}\right)$} & \multicolumn{3}{|c|}{ B:C ratio } \\
\hline & $V_{1}$ & $\mathbf{V}_{2}$ & Mean & $\mathrm{V}_{1}$ & $\mathbf{V}_{2}$ & Mean & $\mathbf{V}_{1}$ & $V_{2}$ & $\begin{array}{c}\text { Mea } \\
\text { n }\end{array}$ & $\mathbf{V}_{1}$ & $\mathbf{V}_{2}$ & Mean & $V_{1}$ & $V_{2}$ & Mean & $V_{1}$ & $V_{2}$ & $\begin{array}{c}\text { Mea } \\
\text { n }\end{array}$ & $V_{1}$ & $V_{2}$ & Mean & $V_{1}$ & $\mathbf{V}_{2}$ & Mean & $V_{1}$ & $V_{2}$ & Mean \\
\hline $\mathbf{N}_{1}$ & 58165.6 & 43291.1 & 50728.4 & 36785.6 & 621911.1 & 29348.7 & 2.05 & 1.02 & 1.54 & 90341.9 & 47690.4 & 69016.2 & 68961.9 & 56467.8 & 62714.9 & 3.56 & 2.31 & 2.93 & 74253.8 & 45490.8 & 59872.3 & 52873.8 & 39189.5 & 46031.6 & 2.81 & 1.67 & 2.24 \\
\hline $\mathbf{N}_{2}$ & 59503.1 & 44120.6 & 51811.9 & 37513.1 & 122130.6 & 29821.9 & 2.04 & 1.01 & 1.52 & 93796.8 & 48548.8 & 71172.8 & 71806.8 & 56533.9 & 64170.4 & 3.60 & 2.24 & 2.92 & 76650.0 & 46334.7 & 61492.3 & 54660.0 & 39332.3 & 46996.1 & 2.82 & 1.62 & 2.22 \\
\hline $\mathbf{N}_{3}$ & 55798.6 & 54620.6 & 55209.6 & 33688.6 & 632510.6 & 33099.6 & 1.86 & 1.47 & 1.66 & 88437.8 & 57883.1 & 73160.5 & 66327.8 & 74099.7 & 70213.7 & 3.33 & 3.02 & 3.18 & 72118.2 & 56251.9 & 64185.0 & 50008.2 & 53305.1 & 51656.7 & 2.60 & 2.24 & 2.42 \\
\hline $\mathbf{N}_{4}$ & 61324.0 & 68511.4 & 64917.7 & 39334.0 & 46521.4 & 42927.7 & 2.12 & 2.12 & 2.12 & 111269.6 & 75001.2 & 93135.4 & 89279.6 & 96775.5 & 93027.6 & 4.39 & 4.07 & 4.23 & 86296.8 & 71756.3 & 79026.6 & 64306.8 & 71648.5 & 67977.6 & 3.26 & 3.09 & 3.17 \\
\hline $\mathbf{N}_{5}$ & 48986.5 & 47628.1 & 48307.3 & 21996.5 & 20638.2 & 21317.3 & 1.15 & 0.76 & 0.96 & 79203.7 & 53595.6 & 66399.6 & 52213.7 & 60686.2 & $\mid 56449.9$ & 2.27 & 1.92 & 2.09 & 64095.1 & 50611.9 & 57353.5 & 37105.1 & 40662.1 & 38883.6 & 1.71 & 1.34 & 1.52 \\
\hline $\mathbf{N}_{6}$ & 68823.9 & 64516.4 & 66670.2 & 43693.9 & 39386.4 & 41540.2 & 2.07 & 1.57 & 1.82 & 111543.9 & 74443.0 & 92993.5 & 86413.9 & 94305.8 & 90359.9 & 3.77 & 3.42 & 3.60 & 90183.9 & 69479.7 & 79831.8 & 65053.9 & 66846.2 & 65950.0 & 2.92 & 2.49 & 2.71 \\
\hline $\mathbf{N}_{7}$ & 48586.1 & 41124.4 & 44855.3 & 28831.1 & 21369.4 & 25100.3 & 1.79 & 1.08 & 1.44 & 77466.3 & 44053.1 & 60759.7 & 57711.3 & 54303.2 & 56007.2 & 3.25 & 2.42 & 2.84 & 63026.2 & 42588.8 & 52807.5 & 43271.2 & 37836.3 & 40553.7 & 2.52 & 1.75 & 2.14 \\
\hline $\mathbf{N}_{8}$ & 46299.0 & 38791.5 & 42545.2 & 26544.0 & 19036.5 & 22790.2 & 1.68 & 0.96 & 1.32 & 76025.3 & 46126.2 & 61075.8 & 56270.3 & 57203.9 & \begin{tabular}{|l|l}
56737.1 \\
\end{tabular} & 3.18 & 2.56 & 2.87 & 61162.2 & 42458.8 & 51810.5 & 41407.2 & 38120.2 & 39763.7 & 2.43 & 1.76 & 2.10 \\
\hline $\mathrm{N}_{9}$ & 45140.7 & 42162.3 & 43651.5 & 24885.7 & 21907.3 & 23396.5 & 1.56 & 1.08 & 1.32 & 75733.0 & 47651.2 & 61692.1 & 55478.0 & 55229.7 & \begin{tabular}{|l}
55353.8 \\
\end{tabular} & 3.07 & 2.39 & 2.73 & 60436.8 & 44906.7 & 52671.8 & 40181.8 & 38568.5 & 39375.2 & 2.32 & 1.74 & 2.03 \\
\hline$N_{10}$ & 39455.9 & 42624.0 & 41039.9 & 19200.9 & 22369.0 & 20784.9 & 1.28 & 1.10 & 1.19 & 80901.0 & 50468.4 & 65684.7 & 60646.0 & 57766.4 & 59206.2 & 3.33 & 2.52 & 2.92 & 60178.4 & 46546.2 & 53362.3 & 39923.4 & 40067.8 & 39995.6 & 2.30 & 1.81 & 2.06 \\
\hline$N_{11}$ & 70451.0 & 69866.3 & 70158.7 & 49926.0 & 49341.3 & 49633.7 & 2.77 & 2.40 & 2.58 & 115067.5 & 74505.5 & 94786.5 & 94542.5 & 100432.7 & 97487.6 & 4.94 & 4.56 & 4.75 & 92759.2 & 72185.9 & 82472.6 & 74734.2 & 73220.3 & 73977.3 & 3.85 & 3.48 & 3.67 \\
\hline$N_{12}$ & 56994.8 & 42944.9 & 49969.8 & 38864.8 & 24814.9 & 31839.8 & 2.48 & 1.37 & 1.92 & 95116.1 & 47037.3 & 71076.7 & 76986.1 & 59642.0 & 68314.1 & 4.58 & 2.96 & 3.77 & 76055.5 & 44991.1 & 60523.3 & 57925.5 & 42228.4 & 50076.9 & 3.53 & 2.16 & 2.85 \\
\hline$N_{13}$ & 44799.8 & 39208.1 & 42003.9 & 26169.8 & 20578.1 & 23373.9 & 1.74 & 1.10 & 1.42 & 74617.7 & 41796.8 & 58207.2 & 55987.7 & 50380.4 & \begin{tabular}{|c|}
53184.0 \\
\end{tabular} & 3.34 & 2.37 & 2.85 & 59708.7 & 40502.5 & 50105.6 & 41078.7 & 35479.3 & 38278.9 & 2.54 & 1.74 & 2.14 \\
\hline$N_{14}$ & 45115.7 & 44215.7 & 44665.7 & 26485.7 & 25585.7 & 26035.7 & 1.76 & 1.37 & 1.56 & 75379.5 & 51369.9 & 63374.7 & 56749.5 & 59461.5 & 58105.5 & 3.38 & 2.86 & 3.12 & 60247.6 & 47792.8 & 54020.2 & 41617.6 & 42523.6 & 42070.6 & 2.57 & 2.12 & 2.34 \\
\hline $\mathrm{N}_{15}$ & 57166.4 & 50308.1 & $\mathbf{5 3 7 3 7 . 2}$ & 38536.4 & 31678.1 & 35107.2 & 2.40 & 1.70 & 2.05 & 109865.5 & 53475.8 & 81670.6 & 91235.5 & 69199.6 & 80217.5 & 5.23 & 3.38 & 4.31 & 83515.9 & 51891.9 & 67703.9 & 64885.9 & 50438.9 & 57662.4 & 3.82 & 2.54 & 3.18 \\
\hline $\mathbf{N}_{16}$ & 69624.7 & 63769.8 & 66697.2 & 48666.3 & 44144.8 & 46405.6 & 2.55 & 2.25 & 2.40 & 113742.3 & 62861.9 & 88302.1 & 94117.3 & 86362.4 & 90239.9 & 4.80 & 4.07 & 4.43 & 91683.5 & 63315.8 & 77499.7 & 71391.8 & 65253.6 & 68322.7 & 3.67 & 3.16 & 3.42 \\
\hline \multirow{2}{*}{ MEAN } & 54764.7 & 49856.4 & & 33820.2 & 28995.2 & & 1.96 & 1.40 & & 91781.74 & 54781.7 & & 70920.5 & 68053.1 & & 3.75 & 2.94 & & \begin{tabular}{|l|}
73273.24 \\
\end{tabular} & 52319.1 & & 52526.6 & 48420.0 & & 2.85 & 2.17 & \\
\hline & S.Em \pm & \multicolumn{2}{|c|}{ C.D at $5 \%$} & S.Em \pm & : C.D a & at $5 \%$ & $\begin{array}{c}\text { S.Em } \\
\pm\end{array}$ & \multicolumn{2}{|c|}{ C.D at 5\% } & S.Em \pm & C.D a & at $5 \%$ & S.Em \pm & \multicolumn{2}{|c|}{ C.D at $5 \%$} & S.Em \pm & \multicolumn{2}{|c|}{ C.D at $5 \%$} & S.Em \pm & \multicolumn{2}{|c|}{ C.D at $5 \%$} & S.Em \pm & \multicolumn{2}{|c|}{ C.D at $5 \%$} & $\begin{array}{c}\text { S.Em } \\
\pm\end{array}$ & \multicolumn{2}{|c|}{ C.D at 5\% } \\
\hline $\begin{array}{l}\text { Varieties } \\
\text { (V) }\end{array}$ & 661.40 & \multicolumn{2}{|c|}{4024.54} & 694.51 & 422 & 26.01 & 0.25 & \multicolumn{2}{|c|}{ NS } & 19148.97 & $\mathbf{N}$ & NS & 4133.06 & $\mathbf{N}$ & & 0.25 & $\mathbf{N}$ & & 9416.08 & & NS & 1703.97 & & NS & 0.25 & & NS \\
\hline$\underset{)}{\text { Nutrients}(\mathbf{N}}$ & 1869.17 & 528 & 7.62 & 1853.56 & 524 & 13.45 & 0.10 & 0. & 30 & 3662.95 & 1036 & 61.95 & 2827.94 & 7999 & & 0.14 & 0.4 & & 2273.49 & 643 & 31.37 & 1831.47 & & 180.9 & 0.10 & & .27 \\
\hline $\mathbf{N}$ at same $\mathbf{V}$ & 2643.41 & 747 & 7.83 & 2621.33 & 7415 & 15.36 & 0.14 & 0. & 42 & 5180.20 & 1465 & 54.01 & 3999.31 & 1131 & 13.45 & 0.21 & 0.6 & & 3215.20 & 909 & 55.33 & 2590.00 & & 326.9 & 0.15 & & .39 \\
\hline $\begin{array}{l}\text { Vat same or } \\
\text { different } N\end{array}$ & 2645.47 & 748 & 3.63 & 2768.5 & 783 & 31.7 & 0.97 & 2 & .7 & 4174.93 & $\mathbf{N}$ & NS & 16038.5 & 4537 & 70.48 & 1.00 & 2.8 & & 36477.10 & 1031 & 188.40 & 6631.10 & & 758.5 & 0.98 & & 2.8 \\
\hline
\end{tabular}

$\mathrm{V}_{1}$ - Vallabh Isabgol- 1

$\mathrm{N}_{3}-\mathrm{RDF}$ FYM + RDF NPK+FeSo 4

$\mathrm{N}_{7}-75 \%$ RDF FYM $+75 \%$ RDF NPK

$\mathrm{N}_{11^{-}} 75 \% \mathrm{RDF} F \mathrm{FY}+75 \% \mathrm{RDF} \mathrm{NPK}+\mathrm{ASP}+$

$\mathrm{PSB}+\mathrm{ZnSO}_{4}+\mathrm{FeSO}_{4}$

$\mathrm{N}_{15}-50 \%$ RDF FYM + 50\% RDF NPK + PSB $\quad \mathrm{N}_{16^{-}} 50 \%$ RDF FYM + 75\% RDF NPK + ASP + PSB +

Vermicompost $\left(1 \mathrm{tha}^{-1}\right), \quad \mathrm{RDFFYM}\left(10 \mathrm{tha}^{-1}\right), \quad \mathrm{ZnSo}_{4}+\mathrm{FeSO}_{4}, \mathrm{~kg} \mathrm{a}^{-1}, \mathrm{PSB} @ 3 \mathrm{~kg} \mathrm{ha}^{-1}$, Azotobacter (AZB)@ $5 \mathrm{~kg}^{-1}, \mathrm{RDF}$
$\mathrm{N}_{1}-$ RDF FYM + RDF NPK $\left(\mathrm{kg} \mathrm{ha}^{-1}\right)$

$\mathrm{N}_{5}$ - Vermicompost $\left(1 \mathrm{t} \mathrm{ha}^{-1}\right)+\mathrm{RDF}$ NPK

$\mathrm{N}_{9-}-75 \%$ RDF FYM $+75 \%$ RDF NPK +ASP

$\mathrm{N}_{13^{-}} 50 \%$ RDF FYM + 50\% RDF NPK + AZB

NS $=$ Non-significant
$\mathrm{N}_{2}-\mathrm{RDF} \mathrm{FYM}+\mathrm{RDF} \mathrm{NPK}+\mathrm{ZnSo}_{4}$

$\mathrm{N}_{6}$ - Vermicompost $+50 \%$ RDF NPK $+\mathrm{ASP}+\mathrm{AZB}$

$\mathrm{N}_{10^{-}}-75 \%$ RDF FYM $+75 \%$ RDF NPK + PSB

$\mathrm{N}_{14}-50 \%$ RDF FYM + 50\% RDF NPK + ASP

DAS $=$ Days after sowing 
The net return was significantly influenced by integrated nutrient management. The maximum net return (Rs. 73,977.3) was recorded as the crop supplied with $\mathrm{N}_{11}(75 \%$ $\mathrm{RD}$ of FYM $+75 \%$ RD of NPK + Azospirillum $\left.+P S B+\mathrm{ZnSO}_{4}+\mathrm{FeSO}_{4}\right)$ which was on par with $\mathrm{N}_{16}(50 \%$ RD of FYM +75 $\%$ RD of NPK + Azospirillum + PSB + $\mathrm{ZnSO}_{4}+\mathrm{FeSO}_{4}$ ) (Rs. 68,322.7), $\mathrm{N}_{4}$ (RD of $\mathrm{FYM}+\mathrm{RD}$ of $\left.\mathrm{NPK}+\mathrm{ZnSO}_{4}+\mathrm{FeSO}_{4}\right)$ (Rs.67,977.6) and $\mathrm{N}_{6}$ (Vermicompost $+50 \%$ RD of NPK + Azospirillum + Azotobacter ) (Rs. 65,950.0). Least net return (Rs. 38,278.9) was recorded as the crop supplied with $\mathrm{N}_{8}$ (Vermicompost + RD of NPK). This was due by saving of $25 \%$ cost of fertilizers resulted more economic yield and which ultimately fetch higher market prices that time resulting more net returns

The interaction effect net return recorded with respect to varieties and integrated nutrient management. The maximum net return (Rs. 74,734.2) was recorded in Vallabh Isabgol-1, supplied with $\mathrm{N}_{11}(75 \% \mathrm{RD}$ of FYM $+75 \%$ $\mathrm{RD}$ of NPK + Azospirillum $+P S B+\mathrm{ZnSO}_{4}+$ $\mathrm{FeSO}_{4}$ ). Least interaction effect (Rs.40,662.1) was observed in Gujarat Isabgol-2, supplied of $\mathrm{N}_{5}$ (Vermicompost + RD of NPK).

This increased net return was due to the fact that the supply of balanced above INM treatments use registered maximum economic yield and which ultimately fetch higher market prices by saving 25\% RD fertilizers cost that time, resulting more net returns. Similar findings were found by researchers like Dwivedi (2008), Nadukeri (2014) in coleus and Shivran et al., (2016a) in Isabgol.

\section{Benefit cost ratio (B: $\mathrm{C}$ ratio)}

The benefit cost ratio (B: $\mathrm{C}$ ratio) was significantly influenced by varieties. Maximum B: C ratio (1: 2.85) was recorded in Vallabh Isabgol-1 followed by $(1: 2.17)$
Gujarat Isabgol-2 maximum B: C ratio was because of higher yield and more market prices fetched during that harvest period (Charak et al., 2005).

The B: C ratio was influenced by integrated nutrient management. Significantly maximum benefit cost ratio (1:3.67) was recorded with application of $75 \% \mathrm{RD}$ of FYM $+75 \% \mathrm{RD}$ of NPK + Azospirillum $+P S B+\mathrm{ZnSO}_{4}+$ $\mathrm{FeSO}_{4}\left(\mathrm{~N}_{11}\right)$ which was on par with 1:3.42 (50 $\%$ RD of $\mathrm{FYM}+75 \% \mathrm{RD}$ of $\mathrm{NPK}+$ Azospirillum $\left.+P S B+\mathrm{ZnSO}_{4}+\mathrm{FeSO}_{4}\right) \mathrm{N}_{16}$, $1: 17$ (RD of FYM + RD of NPK+ $\mathrm{ZnSO}_{4}+$ $\mathrm{FeSO}_{4}$ ) $\mathrm{N}_{4}$ and 1:2.71 (Vermicompost $+50 \%$ $\mathrm{RD}$ of NPK + Azospirillum + Azotobacter) $\mathrm{N}_{6}$ as compared to other treatments combinations by saving $25 \% \mathrm{RD}$ fertilizers cost of fertilizers. Least B: C ratio of $1: 1.52$ was recorded crop supplied with Vermicompost + $\mathrm{RD}$ of $\mathrm{NPK}\left(\mathrm{N}_{5}\right)$.

Because of the use of INM treatments application helps plants to absorb more nutrients leading to higher gross returns, net returns ultimately helps highest benefit cost ratio. These findings are in the line of Venkatesh (2007).

The interaction effects were influenced by the varieties and integrated nutrient management. Maximum B: C ratio (1:3.85) was recorded with Vallabh Isabgol-1 with supply of $75 \%$ $\mathrm{RD}$ of $\mathrm{FYM}+75 \% \mathrm{RD}$ of $\mathrm{NPK}+$ Azospirillum $+P S B+\mathrm{ZnSO}_{4}+\mathrm{FeSO}_{4}$ $\left(\mathrm{V}_{1} \mathrm{~N}_{11}\right)$ on par with Vallabh Isabgol-1, $50 \%$ $\mathrm{RD}$ of $\mathrm{FYM}+75 \% \mathrm{RD}$ of $\mathrm{NPK}+$ Azospirillum $+P S B+\mathrm{ZnSO}_{4}+\mathrm{FeSO}_{4}\left(\mathrm{~V}_{1} \mathrm{~N}_{16}\right)$ (1:3.67) as comparedto other treatment combinations. Least B: C ratio (1:1.34) was recorded in Gujarat Isabgol-2, supplied with Vermicompost + RD of NPK $\left(\mathrm{V}_{2} \mathrm{~T}_{5}\right)$. The increase in $\mathrm{B}$ : $\mathrm{C}$ ratio could be positively correlated to higher gross returns, net returns and reduced cost of inputs campared other treatments (Choudhary et al., 2013). 


\section{References}

Anonymous, 2015b, Horticultural statistics at a glance, state-wise estimates of output from Agriculture and Allied Activities, CSO, MOSPI: 96.

Farooqui, A. A. and Sreeramu, B. S., 2001, A text book an cultivation of medicinal and aromatic crops. Universities Press (India) Ltd., Hyderabad. Pp.168-174.

Sen, S. and Chakraborty, R., 2017, Revival, modernization and integration of Indian traditional herbal medicine in clinical practice importance challenges and future. J. Tradit. and Compliment. Med., 7:234-244.

Hindiholi, M. S., 2006, Effect of dates of sowing and nitrogen levels on growth and yield of Isabgol (Plantago ovata Forsk.). M.Sc. (Hort.) Thesis, Univ. Agril. Sci., Dharwad.

Charak, A. S., Chouhan, G, S. and Sharama, D. D., 2005, Performance of Isabgol (Plantago ovata Forsk.) varieties at varying sowing dates on inceptisals, $J$.
Soil and Crops, 15 (2): 256-259.

Dwivedi, R. S. P., Dwivedi, S. N., Namdeo, K. N., Pathak, S. and Mittoliya, V. K., 2008, Effect of row spacing and nitrogen sources on growth, yield and quality of Isabgol (Plantago ovata Forsk.) varieties. Crop Research, 36 (1, 2 \& 3): 349-353.

Nadukeri, S., Kattimani, K. N. and Kolakar, S. S., 2014, Influence of organic and inorganic fertilizers on growth and tuber yield of Coleus (Coleus forskohlii Briq.) under northern dry zone of Karnataka. Intl. J. Agric. Sci., 10 (1): 119-123.

Shivran, A. C., 2016b, Growth yield and nutrient uptake of Isabgol (Plantago ovata Forsk.) with phosphorus PSB and zinc fertilization. Intl. J. Seed Spices, 6 (1): 66-73.

Venkatesh, N.T. 2007, Integrated nutrient management in Isabgol (Plantago ovate Forsk.). M.Sc (Horti)Thesis, UAS Dharwad.

\section{How to cite this article:}

Siddalingayya V. Salimath, K. N. Kattimani, Y. K. Kotikal, N. S. Nagarja, D. R. Patil, Jameel Jhalegar and Venkatesh, J. 2020. Response of Varieties and Integrated Nutrient Management on Economic Parameters of Isabgol (Plantago ovata Forsk.) under Northern Dry Zone of Karnataka. Int.J.Curr.Microbiol.App.Sci. 9(12): 3068-3072. doi: https://doi.org/10.20546/ijcmas.2020.912.363 